\title{
Antecedentes y lineamientos para el abordaje del sector doméstico
}

\section{Alejandro Balazote}

Universidad de Buenos Aires, Ciudad A. de Buenos Aires, Argentina

Universidad Nacional de Luján, Lujan, Argentina

Correo Electrónico: abalazote336@gmail.com

\section{Sebastián Valverde}

CONICET, Ciudad A. de Buenos Aires, Argentina

Universidad de Buenos Aires. Ciudad A. de Buenos Aires, Argentina

Universidad Nacional de Luján Lujan, Argentina

Correo Electrónico: sebavalverde@yahoo.com.ar

\section{Gabriel Stecher}

CONICET, Ciudad A. de Buenos Aires, Argentina

Universidad Nacional del Comahue, San Martín de los Andes, Neuquén, Argentina

Correo Electrónico: gabrielstecher@gmail.com
Recibido

Octubre de 2018

Aceptado

Marzo de 2019

doi: $10.34096 /$ cas.i49.5305

\section{Resumen}

En este artículo, nos proponemos analizar diferentes antecedentes y lineamientos teóricos del denominado "sector doméstico", "economía doméstica”, "economía campesina” o, genéricamente, los “campesinos". Esta temática de gran relevancia es una de las aristas fundamentales de la antropología económica. Abordaremos estas teorizaciones a partir de la dinámica de las unidades domésticas de los denominados "pobladores" del Parque Nacional Nahuel Huapi, en la región cordillerana de las provincias del Neuquén (departamento Los Lagos) y Río Negro (departamento Bariloche), en la región norte de la Patagonia argentina. A partir del análisis de estos procesos junto con los diferentes antecedentes teóricos, formularemos nuestra propuesta para el abordaje del sector doméstico. Esto incluye la perspectiva etnográfica característica de la antropología y las variables estructurales (junto con otras específicas) que afectan a estas poblaciones en particular.

\section{Theoretical background and guidelines for approaching the household sector}

\begin{abstract}
This article analyzes the theoretical background and guidelines behind the concepts of "household sector", "household economy", "peasant economy" or, in general, "peasantry", all of which remain at the core of Economic Anthropology. We approach
\end{abstract}

\section{Palabras clave}

Sector Doméstico; Economía Doméstica; Campesinos; Antropología Económica; Parque Nacional Nahuel Huapi

\section{Key words}

Household Sector, Household Economy, Peasants, Economic Anthropology; Nahuel Huapi National Park 
these theoretical conceptualizations in the light of domestic units' dynamics among the so-called "dwellers" [pobladores] of the Nahuel Huapi National Park, in the Andean region of northern Patagonia, in the Argentinian provinces of Neuquén (Los Lagos Department) and Río Negro (Bariloche Department). Through relating the analysis of these processes to different theoretical backgrounds, we articulate our own proposal for addressing the household sector. This includes the ethnographic perspective, which characterizes Anthropology, while accounting for structural as well as specific variables that affect these particular groups.

\section{Antecedentes e diretrizes para a abordagem do setor doméstico}

\section{Resumo}

Palavras-chave

Setor Doméstico; Economia Doméstica; Camponeses; Antropologia Econômica; Parque Nacional Nahuel Huapi
Neste artigo, propomos analisar diferentes antecedentes e orientações teóricas do chamado "setor doméstico", "economia doméstica”, "economia camponesa" ou, genericamente, os “camponeses”. Essa temática de grande relevância é uma das arestas fundamentais da antropologia econômica. Abordaremos essas teorizações a partir da dinâmica das unidades domésticas dos chamados "moradores" do Parque Nacional Nahuel Huapi, na região das cordilheiras das províncias de Neuquén (departamento Los Lagos) e Río Negro (departamento Bariloche), na região norte da Patagónia Argentina. A partir da análise desses processos, juntamente com os diferentes fundamentos teóricos, formularemos nossa proposta de abordagem do setor doméstico. Isso inclui a perspectiva etnográfica característica da antropologia e as variáveis estruturais (juntamente com as variáveis específicas) que afetam essas populações em particular.

\section{Introducción}

La problemática del denominado "sector doméstico", "economía doméstica", "comunidad doméstica", "modo de producción doméstico", "economía campesina” o, genéricamente, "los campesinos", posee gran relevancia y es una de las temáticas fundamentales de la antropología económica.

En este artículo - en el marco del presente dossier de antropología económica- nos proponemos analizar los diferentes lineamientos teóricos retomando antecedentes en relación con esta temática, que se han dado tanto en el mundo en general como en América Latina y la Argentina en particular. Los vincularemos con diversas realidades específicas abordadas por diferentes investigaciones de los autores. Nos proponemos dar cuenta de la dinámica de las unidades domésticas de los denominados "pobladores" del Parque Nacional Nahuel Huapi, en la región cordillerana de las provincias del Neuquén (departamento Los Lagos) y Río Negro (departamento Bariloche), en la región norte de la Patagonia argentina.

Efectuaremos un breve recorrido de las trayectorias de estas poblaciones a lo largo del siglo XX y lo que va del XXI. Nos detendremos especialmente en la década de 1930 - momento en que se conformó esta área protegida, hecho que provocó profundas consecuencias para dichas poblaciones- Luego, nos centraremos en la década de 1960, cuando se acentuaron las migraciones hacia las incipientes localidades de la región, como Villa la Angostura y San Carlos de Bariloche, lo que dio como resultado la desestructuración y redefinición de muchas de las unidades domésticas. Finalmente, nos 
enfocaremos en las últimas décadas, cuando muchas de estas familias ya asentadas en las ciudades comenzaron y/o profundizaron su autorreconocimiento y visibilización pública como parte del pueblo indígena mapuche. Esto plantea, al mismo tiempo, un retorno a los territorios ancestrales.

A la vez, en este contexto, a partir de los vínculos familiares, se da un proceso de reetnización, reemergencia étnica y retorno a los territorios ancestrales o "viajes de vuelta", como los ha caracterizado João Pacheco de Oliveira (1999). Por ello, también nos referiremos al lugar del ámbito familiar en la transmisión de aspectos socioculturales.

Por último, a partir del análisis de estas situaciones, en conjunción con los diferentes antecedentes teóricos, formularemos una serie de lineamientos para el abordaje del sector doméstico. Esto incluye la perspectiva etnográfica característica de la antropología y las variables estructurales (junto con otras específicas) que afectan a estas poblaciones en particular.

Para el desarrollo de este ensayo, hemos recopilado y sistematizado diferentes antecedentes teóricos en relación con la antropología económica, el denominado "sector doméstico" - tanto en la Argentina como en el mundo-y las poblaciones originarias del norte de la Patagonia. Para el abordaje de las poblaciones de esta región, empleamos diferentes fuentes documentales e incluimos los resultados de entrevistas efectuadas entre 2000 y 2018 a integrantes y dirigentes de las comunidades mapuches, así como a diversos sectores e instituciones involucradas. La inclusión de esta zona se basa en el trabajo que desde hace años venimos desarrollando, no solo a partir de investigaciones, sino también como resultado de diferentes proyectos de transferencia y/o extensión, llevados a cabo en forma conjunta con diversas comunidades de la zona, desde principios de la década del 2000 desde el ámbito de la Universidad de Buenos Aires, la Universidad Nacional del Comahue, la Universidad Nacional de Luján y el Consejo Nacional de Investigaciones Científicas y Técnicas (CONICET). En efecto, aquí se ven plasmados los resultados de años de investigaciones desarrolladas por los tres autores. El primero de ellos es antropólogo social, profesor titular de la asignatura Antropología Rural y de la materia Antropología Económica de la Facultad de Filosofía y Letras de la Universidad de Buenos Aires. Desde la década de 1980, investiga estas temáticas en relación con pequeños productores rurales e indígenas, en especial, del pueblo mapuche de Norpatagonia. El segundo autor, como antropólogo social investiga desde hace años la conflictividad territorial del pueblo mapuche en relación con los procesos de actualización étnico-identitaria, las transformaciones socioeconómicas regionales y el desarrollo de los movimientos indígenas. Por último, el tercero es técnico forestal (como formación de grado) y se formó en Estudios Sociales Agrarios (de posgrado). Se ha desempeñado en diferentes proyectos de intervención en la región con pobladores e indígenas desde hace veinte años e investiga sobre estas dinámicas.

\section{Antecedentes teóricos: el denominado "sector doméstico" o "eco- nomía doméstica"}

En relación con estos temas, son muy conocidos los aportes del agrónomo ruso Alexander V. Chayanov (1974), que pertenece a la denominada "Escuela para el análisis de la organización y producción campesinas". Sus investigaciones se desarrollaron a la luz de los intensos debates que tuvieron lugar en Rusia sobre la cuestión agraria a fines del siglo XIX y principios del XX.

El autor diferencia "familia" de "unidad" o "grupo doméstico" y señala que "es indudable que el concepto de familia, particularmente en la vida campesina, pocas veces coincide con el concepto biológico que lo subyace y en su contenido intervienen una 
serie de complicaciones económicas y domésticas" (Chayanov, 1974, p. 48). Siguiendo con esta argumentación:

los estadísticos del zemstvo ruso, por ejemplo, al realizar censos en los hogares establecieron que para el campesino el concepto de familia incluye a las personas que comen siempre en la misma mesa o que han comido de la misma olla. (Chayanov, 1974, p. 48)

El mismo autor ha caracterizado al campesinado como un tipo específico de economía, cuya actividad está determinada por las necesidades del grupo doméstico. Por ello, ha teorizado acerca de la relación entre el balance y el consumo, que condiciona el esfuerzo productivo de la familia a lo largo de su ciclo de vida. Chayanov (1974) se centró en la importancia de satisfacer las necesidades familiares, las cuales van cambiando a lo largo del ciclo familiar. Uno de los aportes fundamentales de este destacado teórico es la especificidad de estas economías, a las cuales los conceptos de "renta" y "salario", por lo tanto, no son aplicables.

Las tesis de Chayanov soslayaron en su análisis la importancia de factores que explican el surgimiento de la economía campesina, su permanencia y recomposición, más allá de los mecanismos demográficos. Su preocupación fundamental fue analizar la unidad familiar desde la perspectiva micro y sus diferenciaciones en función del ciclo de desarrollo; de ahí que a esta teoría se la conozca como de diferenciación demográfica.

Este autor de principios del siglo XX mantuvo una serie de debates con pensadores marxistas contemporáneos. Para estos teóricos —en el contexto de la revolución Rusa de 1917 y el proceso de transformación social en su conjunto-, el foco principal estaba puesto en la manera en que el avance de las relaciones capitalistas de producción establecía diferencias entre los campesinos, que obligaban a unos a vender su fuerza de trabajo, mientras otros se capitalizaban y contrataban asalariados (Balazote y Radovich, 1992; Schiavoni, 1998). De modo que estas lecturas son conocidas como de diferenciación social.

En lo que respecta a la disciplina antropológica, el estudio de los campesinos fue abordado inicialmente en términos de "contenidos culturales", mediante los denominados "estudios de comunidad". El continuum folk-urbano de Redfield en las décadas de 1940 y 1950 tendió a ligar a los campesinos con lo primitivo. Desde esta lectura, los campesinos y las sociedades primitivas comparten el hecho de vivir en sociedades pequeñas, aisladas, autosuficientes, culturalmente homogéneas, que registran cambios lentos, poseen un grado mínimo de división del trabajo y para producir utilizan tecnología sencilla. En lo que respecta a su organización social, estos grupos se basan en el parentesco y el compadrazgo; las motivaciones económicas están ausentes (Schiavoni, 1998).

Estas lecturas recibieron críticas de diferentes autores que advirtieron en ellas una mirada sesgada y simplista de tales aproximaciones, ya que fueron soslayados otros aspectos del análisis, como la relación con diversos sectores sociales regionales y nacio-

1. Un ejemplo es el de Oscar Lewis, que reestudió Tepoztlán (México), la zona que Redfield había investigado, y encontró conflicto donde este autor, dos décadas antes, había descripto armonía y heterogeneidad social donde aquel había presentado una supuesta homogeneidad, que no era tal (Roseberry, 1989). nales en que dichos campesinos se insertan. ${ }^{1}$ Por ello, se fueron elaborando diversos aportes alternativos.

Para continuar con las contribuciones efectuadas desde la antropología -específicamente desde la antropología social-, la organización social de las economías campesinas fue abordada por Raymond Firth (1976). En su conocida definición de "campesino", asimila esta categoría a la de un productor de pequeña escala e incluye como economías campesinas a los pescadores, artesanos, etc. Los trabajos de Firth forman parte de los nuevos estudios sobre campesinos en un área nueva (por aquel entonces) de las ciencias 
sociales, que empezó a destacarse en la década de 1960, cuando comenzaban a editarse publicaciones específicas referidas a esta problemática.

La antropología social británica retomó diversas cuestiones que ya estaban presentes en el análisis de Chayanov. De allí el interés de la obra de este autor ruso cinco décadas después de ser publicada y traducida al inglés y, luego, a otros idiomas. ${ }^{2}$ Dentro de esta preocupación por el ciclo de desarrollo del grupo doméstico, Meyer Fortes (en Balazote y Radovich, 1992) diferenció tres fases: "expansión", "fisión" y "reemplazo".

\section{La primera comienza con el matrimonio y finaliza cuando declina el ciclo de fertilidad femenino. La fisión es la que engloba a los matrimonios de los hijos culminando al casarse el último. Finalmente, la etapa de reemplazo, concluye con la muerte de ambos progenitores o cuando estos abandonan la actividad productiva. (Balazote y Radovich, 1992, p. 34)}

También es necesario destacar los aportes de Marshall Sahlins (1972) en la década de 1970, a partir de una lectura culturalista y particularista. Este autor norteamericano permitió situar a las unidades domésticas en un contexto más amplio, al plantear la existencia - a partir de las funciones propias de la política- de las relaciones de reciprocidad y redistribución. El autor problematizó las dinámicas resultantes de diferentes unidades domésticas articuladas entre sí -la denominada "comunidad doméstica" en la que el rol de los jefes resulta fundamental.

La perspectiva de Sahlins posibilitó, por un lado, redescubrir a Chayanov y favorecer una lectura más antropológica de este autor; pero a la vez, facilitó la reflexión acerca de las diversas interrelaciones en el seno de un contexto más amplio que el de las unidades domésticas individuales. La mencionada "comunidad doméstica" definida por Sahlins (1972) constituye, precisamente, esa entidad más abarcadora.

Sin desmerecer estos aportes, debemos señalar las agudas críticas que recibió Sahlins (y que los autores de este texto hacemos propias) al no visualizar claramente el marco regional, la dominación colonial y hasta qué punto parte de la "modalidad doméstica" que él caracterizaba ya se estaba transformando por la expansión capitalista en la época en que el autor desarrollaba sus teorizaciones o retomaba a otros autores.

Luego, en la década de 1970, se realizaron estudios encuadrados en las lecturas de Marx -efectuadas, principalmente, en Francia- que dieron cuenta de las "economías domésticas" en un contexto más amplio. El concepto teórico de "modo de producción", que echa luz acerca de la noción de "totalidad social", o bien su aplicación a realidades empíricas concretas, como la de "formación económico-social", se convirtieron en herramientas analíticas para abordar el sector (o el modo de producción) doméstico, pero ya privado de sus condiciones de reproducción social, subordinado al modo de producción capitalista. ${ }^{3}$

En América Latina, los trabajos de Steward primero, y luego, los de uno de sus discípulos, Eric Wolf ${ }^{4}$ (1971), desplazaron el interés por los contenidos culturales hacia el abordaje de las relaciones estructurales que ligan a los campesinos con el sistema social englobante (Schiavoni, 1998). El surgimiento de este enfoque (dentro del cual también se destacaron, posteriormente, William Roseberry, 1991; Sidney Mintz, 1996, entre otros $)^{5}$ se vinculó con una tendencia dentro de la antropología que buscaba, precisamente, romper con los estudios de comunidad de Redfield, y que tendía a estudiar a las sociedades nacionales no como comunidades aisladas, sino como un todo (Comas D’Argemir, 1998; Balazote, 2007).
2. La obra principal de Chayanov, La organización de la unidad económica campesina, fue publicada en Moscú en 1925. Si bien recién fue traducida al inglés en 1966 (Schiavoni, 1998), en América Latina y en Argentina particularmente, la obra fue descubierta en la década de 1970.
3. Caben destacar los aportes de Claude Meillassoux (1993), en especial en la obra Mujeres, graneros y capitales, en la que analiza la interrelación entre los países centrales y los periféricos excolonias y, a la vez, entre las ciudades y los ámbitos rurales. También cabe destacar el estudio de Ann Stoler (1987) sobre las plantaciones de Sumatra.

4. Para un análisis detallado de esta escuela, ver el artículo de Alejandro Balazote: “Consideraciones en torno a la Economía Política" (2007). También ver el capítulo, autoría de Dolors Comas D’Argemir (1998) “Economía Política y Antropología Económica", que forma parte de la obra Antropología Económica.

5. En España cabe destacar los aportes de Dolors Comas D’Argemir, (1998) y Susana Narotzky (2004) en esta línea. 
6. No nos detendremos en este aspecto, ya que trasciende los objetivos de este artículo. En relación con la emergencia y el desarrollo de una serie de investigaciones encuadradas en la antropología social, se pueden consultar los trabajos de Ratier (2010) y Herrán (1990). En relación con la antropología económica, ver Balazote (2007).
$\mathrm{Al}$ considerar el marco regional y estas interrelaciones con diversos agentes, se profundizaron los estudios que revisaban los diferentes "tipos" de campesinos, en función de sus formas diferenciales de inserción en la sociedad mayor (Schiavoni, 1998).

En términos generales, las investigaciones sobre el campesinado latinoamericano se han centrado en la relación con el capitalismo, respecto de lo cual pusieron el acento sobre la subordinación cultural, económica y política de la pequeña producción familiar al sector capitalista y terrateniente.

En la Argentina, tuvo lugar una destacada producción que recuperó esos planteos y analizó el campesinado retomando las formulaciones que vinculan a estos sectores sociales con la sociedad global. Quienes han abordado el estudio del agro en el contexto local coinciden en señalar que la gran mayoría de las explotaciones en la Argentina no admiten la caracterización en términos de "unidades campesinas", y por eso se ha preferido utilizar, en mayor medida, la noción de "pequeños productores".

Estos tempranos aportes fueron los primeros estudios efectuados desde la perspectiva de la antropología social y la antropología rural, esta última no ya desde la tradición folklórica, sino como subdisciplina de la primera. Esto ocurrió hacia la década de 1970, a medida que se consolidaba la disciplina, y en particular esta línea de la antropología en nuestro país entre las primeras generaciones de egresados de la carrera (creada en 1958). ${ }^{6}$

Entre ellos, podemos mencionar a Hebe Vessuri (1976) y Santiago Bilbao (1964) para la zona de Santiago del Estero y Tucumán; Leopoldo Bartolomé (1975) para Misiones; y Esther Hermitte y Carlos Herrán (1977) para Catamarca. Por su parte, Eduardo P. Archetti y Kristi Anne Stølen (1975) han trabajado en relación con los campesinos de una localidad del norte de Santa Fe, cuyo nombre de fantasía es "Santa Cecilia". Estos autores efectúan la distinción que ya hemos señalado entre "familia" y "grupo doméstico". Por "familia" entienden "[...] un sistema de relaciones sociales basado en el parentesco que regula el conjunto de derechos y obligaciones sobre la propiedad" (Archetti y Stølen, 1975, pp. 50-51). Por "grupo doméstico" definen: "un sistema de relaciones sociales que basado en el principio de residencia común, regula y garantiza el proceso productivo" (1975, p. 51). No es necesario que la totalidad de los miembros de una familia integren un mismo grupo doméstico, o que todos los integrantes de un grupo doméstico estén vinculados por relacionales de parentesco (Archetti y Stølen, 1975; Balazote y Radovich, 1992). Paralelamente, estas conceptualizaciones elaboradas en la Argentina retomarían muchos antecedentes de otras geografías, pero adaptadas al contexto local. Tal es el caso de las fases de desarrollo del ciclo doméstico, respecto del cual Archetti y Stølen (1975), en su análisis de los colonos de Santa Cecilia en el norte de Santa Fe, perfeccionaron el modelo de Fortes e incluyeron subfases dentro de cada una de ellas, tomando como variable las edades de los hijos (dado su potencial laboral) (Balazote y Radovich, 1992).

Tomando en cuenta estos antecedentes, gran cantidad de trabajos realizados en la Argentina han dirigido su atención al análisis de las diferentes tipologías, en gran medida, a partir de la interrelación con el contexto social más amplio.

En forma paralela, debemos mencionar cómo, a partir de la reinstauración de la democracia en la Argentina, en la década de 1980 - y junto con la consolidación de diferentes equipos profesionales - se publicarían una serie de trabajos que abordan, de entre las diversas temáticas de la antropología económica, precisamente la que aquí venimos desarrollando. Es el caso de la obra Producción doméstica y capital. Estudios desde la Antropología Económica (Trinchero, 1995) y de otras compilaciones o dossiers de esta misma revista, Cuadernos de Antropología Social, coordinados por este autor, 
profesor titular de la asignatura homónima en la Facultad de Filosofía y Letras de la Universidad de Buenos Aires. ${ }^{7}$

Para finalizar, cabe destacar que, durante los últimos años, se han desarrollado estudios referidos al lugar de la mujer en la unidad doméstica (Federici, 2013), que se enmarcan en los crecientes estudios de género en antropología, sociología y otras disciplinas sociales. Este crecimiento coincide con el desarrollo y la consolidación de diferentes colectivos y reivindicaciones específicas. No profundizaremos en este aspecto, pero sí deseamos mencionarlo en este apartado y destacar que esta problematización resulta, desde ya, una tarea pendiente a desarrollar en aportes futuros.

\section{Los pobladores mapuches del Parque Nacional Nahuel Huapi}

La dinámica inherente a los pequeños productores con que ejemplificaremos este artículo se refiere a los denominados "pobladores" ${ }^{8}$ (en muchos casos, se trata de indígenas del pueblo mapuche) en la zona del Parque Nacional Nahuel Huapi, en el norte de la Patagonia argentina.

El sometimiento militar de este pueblo ${ }^{9}$ tuvo lugar a fines del siglo XIX, cuando se llevaron a cabo las campañas - de carácter genocida - conocidas eufemísticamente como "Conquista del desierto" (en La Pampa y la Patagonia, entre 1879 y 1885). Al mismo tiempo, en el occidente cordillerano (actual Estado chileno) se desarrollaba una operación militar similar (denominada "Pacificación de la Araucanía") (Radovich y Balazote, 2009). La "Conquista del desierto" trajo como consecuencia el exterminio y sometimiento de miles de indígenas, pero también la privatización y concentración de grandes extensiones de tierra necesarias para la expansión de la clase terrateniente y la consolidación del modelo agroexportador. Otro efecto fue el reasentamiento de la población sobreviviente en tierras marginales, donde adoptaron como actividad económica preponderante la crianza de ganado menor (ovino y caprino) en forma extensiva (Radovich y Balazote, 2009).

Luego de las conquistas y con el proceso de conformación del Estado-nación, las políticas buscaron permanentemente homogeneizar, en términos culturales, a la población asentada en el territorio, tanto a los indígenas como a otros grupos sociales considerados "indeseables" (como inmigrantes chilenos de bajos recursos, muchas veces descendientes de indígenas y criollos).

Podemos definir a los pobladores asentados en dicho parque nacional como "pequeños productores" centrados en la unidad doméstica como ámbito de producción, consumo y reproducción física, biológica y sociocultural (Archetti y Stølen, 1975; Balazote y Radovich, 1992). Durante aquellos años, la actividad de estos grupos ha sido la agricultura a pequeña escala, fundamentalmente la siembra de cereales, frutales y hortalizas que se destinan, en gran parte, al autoconsumo y al intercambio de excedentes. También se dedican a la cría de ganado (ovino, caprino y bovino) y a la comercialización de diferentes productos derivados de estas actividades. Por ello, es posible caracterizarlos como "pequeños crianceros", ya que desarrollan un manejo del ganado sumamente singular de este tipo de pequeños productores familiares, de tipo trashumante. $^{10}$

En 1934, en las adyacencias del lago Nahuel Huapi se conformó el parque nacional homónimo - primera área protegida de Sudamérica con sede en la ciudad de San Carlos de Bariloche-. Desde su fundación, la Administración de Parques Nacionales ha ejercido un rol clave en el desarrollo regional, ya que se transformó en el principal referente del Estado sobre el territorio. Es responsable del aprovisionamiento de
7. Estos antecedentes en la antropología económica local se analizan con mayor detalle en el dossier de Cuadernos de Antropología Social, 26, del año 2007 (Trinchero, Balazote y Valverde, 2007).

8. La denominación englobadora de "pobladores" fue definida desde las políticas estatales e internalizada por los propios sujetos como forma de designarse a sí mismos y a otros. Se refiere a que es "oriundo" del lugar, pequeño productor, y que basa su subsistencia en una economía familiar. Esta categoría de "pobladores" representa un universo heterogéneo que, en algunos casos, posee una raíz cultural común con los indígenas (no así en otros casos, que son de origen criollo o, en menor medida, europeo) (Valverde, García y Bersten, 2008).

9. El pueblo mapuche ("gente de la tierra" en su lengua originaria, el mapudungún) es uno de los grupos originarios más importantes de la Argentina, aunque la gran mayoría de ellos reside en Chile. En dicho país, es el pueblo indígena más significativo: suman un millón de integrantes (Juliano, 1996). Están asentados en la Octava, Novena y Décima Región (en el sur) y en la región Metropolitana (capital, en la zona centro) como resultado de las migraciones. En la Argentina, también es el pueblo más numeroso (más de 200.000 miembros), pero están más atomizados, junto con otros grupos originarios, como los qom, kollas, diaguitas, etc. (INDEC, 2012). El pueblo mapuche se asienta en la Patagonia (la zona más austral de América del Sur), en las provincias del Chubut, Río Negro, Neuquén, La Pampa y Santa Cruz, en la provincia de Buenos Aires (Radovich y Balazote, 2009) y, como resultado de las migraciones, en el Área Metropolitana de Buenos Aires (Ciudad de Buenos Aires y Gran Buenos Aires).

10. La trashumancia consiste en el traslado de los animales en función de la época del año, del ámbito de la denominada "veranada" (zona alta de las montañas) a la "invernada” (zonas bajas cerca de las poblaciones y de la costa de los lagos) donde pastan los animales cuando en las zonas altas hay precipitaciones (en forma de lluvia y nieve). 
infraestructura básica (por ejemplo, de edificios públicos y privados), loteó tierras, asignó autorizaciones para la instalación de pobladores y fijó los controles para el conjunto de los recursos (permisos de caza, tala de árboles, extracción de leña, etc.) (Valverde et al., 2008).

A lo largo del siglo XX, los integrantes de las diversas familias de pobladores asentados en las áreas rurales cercanas a las localidades de Bariloche y Villa la Angostura (al igual que las restantes urbes de la región) fueron migrando hacia estas ciudades para desempeñarse en diferentes trabajos asalariados - ya sea diariamente, por temporada o en forma permanente-.

Las actividades agrícolo-ganaderas que estos grupos desarrollaban en los ámbitos rurales fueron declinando como consecuencia de la acentuada reducción de los espacios territoriales ante el avance de diferentes agentes estatales y privados; las crecientes restricciones al uso productivo; en algunos casos, los procesos de expulsión forzada desde diversos ámbitos rurales (principalmente por parte de Parques Nacionales); la imposibilidad que tenían las economías domésticas de acrecentar su producción al tiempo que se incrementaban sus integrantes; y la progresiva retracción del mercado para la venta de las diversas elaboraciones domésticas de las que antes eran abastecedores. Esto los llevó a migrar estacionalmente primero, y luego, de manera permanente, hacia zonas rurales periurbanas o ámbitos urbanos.

A partir del análisis de estas páginas, observamos, pues, que para comprender la dinámica de estos grupos domésticos, resulta insoslayable atender al contexto más amplio en que se insertan y la subordinación a diversos agentes económicos y políticos regionales, acorde con los antecedentes teóricos (que señalamos en el apartado anterior).

Para comprender las expulsiones del medio rural a las que antes aludíamos, es necesario considerar que esta área protegida, al asentarse en la región cordillerana, es de las más valoradas (ya que cuenta con lagos, montañas y atractivos paisajes). Esto explica que en la segunda mitad de la década de 1950, y en especial en la década de 1960, empiece a advertirse una creciente conflictividad entre los pobladores indígenas y criollos (de escasos recursos) con Parques Nacionales y diversos agentes privados con intereses ligados a la explotación turística e inmobiliaria.

En el ámbito urbano, los pobladores migrantes se fueron asentando en los barrios característicos de los sectores populares, con menores y peores servicios. Progresivamente, se fueron empleando en trabajos, por lo general, precarios e informales, característicos de los sectores pauperizados: el servicio doméstico (las mujeres) y la construcción (los hombres), además de diversas labores vinculadas a los servicios, en gran medida del mercado turístico (transporte, jardinería, servicios gastronómicos, etc.), muchas de ellas de carácter informal y temporario; por ello, generalmente, reciben bajas remuneraciones y padecen mayor precariedad laboral que la población no indígena. En algunos casos, fueron logrando insertarse en diferentes empleos en al ámbito estatal (por lo general, más formales y estables).

Históricamente, entre estos migrantes que fueron estableciéndose desde hace varias décadas en Bariloche, Villa la Angostura y otras ciudades, se dio un proceso de "desadscripción étnica", de ocultamiento de la identidad, a partir de la internalización —en ellos mismos- de los prejuicios de vastos sectores sociales en relación con los pueblos indígenas (Radovich, 1992).

No obstante, en muchas ocasiones fue posible seguir transmitiendo en forma oral —de generación en generación-diferentes prácticas culturales del pueblo mapuche en relación con diversos aspectos, como las tareas rurales, la confección de tejidos, la 
elaboración de comidas y un conjunto de "costumbres" asociadas a lo "rural" o a ciertas características locales; pero otras veces, estas prácticas culturales no eran transmitidas a las nuevas generaciones - o sí, pero de manera muy fragmentaria- dado el racismo de que eran objeto estas poblaciones. Tal es el caso de la enseñanza de la lengua mapuche - el "mapudungún" - que los mayores hablaban pero no enseñaban a las nuevas generaciones, para protegerlas del racismo y la persecución. Esta política coercitiva - claramente encuadrable dentro de la categoría de "etnocidio" $-{ }^{11}$ resultó sumamente exitosa, dado que, en la actualidad, son escasos los habitantes de las distintas comunidades que la hablan (Radovich y Balazote, 2009).

Sin embargo, muchas de estas prácticas culturales del pueblo mapuche se pudieron mantener y transmitir a los integrantes más jóvenes, a veces disimuladas bajo el culto católico. ${ }^{12} \mathrm{El}$ hecho de que estas prácticas culturales estuvieran presentes y de que se mantuvieran presentes y vivas en la memoria colectiva es clave para comprender la revalorización de la identidad y de los saberes ancestrales.

De esta forma, comprendemos que, en las últimas décadas, los hijos y nietos de aquellos desplazados que residen en el medio urbano hayan iniciado un proceso de revalorización de la identidad mapuche. Nacidos y criados o llegados a corta edad a la ciudad lograron un mejor nivel de instrucción formal y capacitación laboral que sus mayores y pasaron, además, por experiencias en sindicatos, partidos políticos y comunidades de base de la Iglesia católica (Radovich, 1992).

En contraste con las políticas homogeneizadoras que tendían a negar la diversidad, predominantes durante el siglo XX, en la década de $1970^{13}-\mathrm{y}$, con mayor énfasis, con el retorno de la democracia, en la década de 1980-, comenzaron a originarse diversas organizaciones indígenas a partir de esta dirigencia surgida en los ámbitos urbanos, que fueron instalando la "cuestión indígena" en la agenda pública y lograron un reconocimiento por parte de entes estatales y no estatales. A la vez, estos procesos se sustentan y retroalimentan en las crecientes dinámicas de "reemergencia", "reactualización de la identidad", "transfiguración étnica" (Ribeiro, 1971; Bartolomé y Barabas, 1996) o "revival de lo étnico" (Vázquez, 2000).

Así, diversos grupos familiares que residen en las localidades de la zona comenzaron a adscribirse y reivindicarse públicamente como "mapuches" y demandaron al Estado su formalización como "comunidades" ${ }^{14}$ Estos procesos de "reterritorialización" -o "viajes de vuelta", como ha señalado Pacheco de Oliveira (1999) — en muchos casos, implicaron el retorno a ámbitos de los que habían sido expulsados o que debieron abandonar en el pasado; o bien supusieron una reafirmación (no ya como "pobladores", sino como mapuches) en los ámbitos donde habían estado asentados tradicionalmente. En algunos casos, se nuclearon en torno a un poblador titular de un Permiso Precario de Ocupación y Pastaje (PPOP) que otorgaba Parques Nacionales a los pobladores asentados en su jurisdicción antes del inicio de sus funciones, en 1937. En otros, no eran beneficiarios de estos permisos y habían sido desalojados. En estos procesos, ha sido definitoria la manera en que los integrantes de estas familias - devenidos en la nueva dirigencia indígena urbana - revalorizaron su propia cultura en este contexto no tradicional (Valverde, 2010). Son dinámicas comparables a las verificadas en toda América Latina, que implicaron la resignificación de "campesinos a indígenas" (Bengoa, 2009) y que, en la Patagonia, podemos resumir como de "pobladores a mapuches" (Valverde, 2010).

Para explicar estos procesos, debemos comprender cómo, en el ámbito urbano, continuaron vinculándose las diversas familias emparentadas entre sí, asociadas a un territorio, a una historia, a sus antepasados, a ciertos "hitos" que fueron elaborando, reiterando y transmitiendo estos aspectos, y revitalizándose a partir de las nuevas
11. Pierre Clastres ha definido como "etnocidio" a "la destrucción sistemática de los modos de vida y de pensamiento de gentes diferentes a quienes Ilevan a cabo la destrucción" (1996, p. 56).

12. Es el caso de la denominada "Fiesta de San Juan", que se lleva a cabo el 24 de junio, celebración religiosa de la fe cristiana que fue popularizada por parte de la población indígena, ya que el día cristiano de San Juan coincide con el "Wiñoy Xipantu” (el año nuevo del pueblo mapuche) (Valverde et al., 2008).

13. El pueblo mapuche conformó, en esta región, una de las primeras organizaciones indígenas del país - la “Confederación Indígena Neuquina"- (luego denominada hasta nuestros días "Confederación Mapuche Neuquina”). Con el retorno de la democracia, surgió “Nehuén Mapu" ('la fuerza de la tierra'), en la ciudad de Neuquén y también se conformaron los "Centros Mapuche" (de diversas localidades de la provincia, entre ellas San Carlos de Bariloche) y el "Consejo Asesor Indígena", ambos en la provincia de Río Negro.

14. De acuerdo con los datos del censo de 2001, en la ciudad de Bariloche, en un 10,2\% de los hogares, al menos una persona se ha reconocido como perteneciente o descendiente de un pueblo indígena. Una proporción similar encontramos en Villa la Angostura (12,1\%) (INDEC, 2001 - Reprocesamiento propio de la Base de datos "Redatam+SP" disponible en sitio web). 
generaciones (en otros contextos). Remarcamos, en este sentido, la importancia del grupo doméstico como transmisor de normas y valores, tal como señalábamos en un pionero trabajo: "el carácter reproductivo de las unidades domésticas implica una determinada forma de concebir el mundo, de relacionarse con la naturaleza, de los hombres entre sí, de organizarse social y políticamente y de ejercer la vida cotidiana" (Balazote y Radovich, 1992, p. 28).

De modo que los flujos en sentido rural-urbano a los que aludíamos no implicaron el fin de los fluidos vínculos entre los integrantes que fueron instalándose progresivamente en las ciudades y sus familiares que permanecieron en el campo. Por el contrario, la reproducción de interrelaciones con el territorio es expresión de la importancia de este como parte fundamental de la identidad del grupo, lo que a su vez permite comprender las crecientes movilizaciones en defensa de aquellos territorios.

\section{Propuesta para el abordaje del sector doméstico y palabras finales}

Uno de los debates fundamentales que se han dado en la antropología económica (que formulamos al inicio de este trabajo) es la relación entre estas poblaciones particulares con procesos y condicionamientos de la macroestructura. Analizamos los destacados aportes de Chayanov (1974) para comprender la dinámica demográfica de las familias campesinas, pero también cómo luego surgieron diferentes lecturas que plantearon unidades de análisis más amplias y la interrelación de dichas unidades entre sí (Sahlins, 1972) o bien con diversos sectores económicos que las condicionan (Wolf, 1971). Repasamos también las lecturas efectuadas en la Argentina y cómo han estado centradas en la relación entre estas poblaciones particulares con diversos agentes más amplios, políticos y económicos, que las condicionan.

El análisis que hemos efectuado se refiere al denominado "sector doméstico" o "economía doméstica" y lo ejemplificamos a partir de los pobladores del Parque Nacional Nahuel Huapi. Una propuesta que retomamos es la de Rosberry (1989), ya que entendemos que plantea muy adecuadamente la incidencia de procesos más generales junto con otros específicos que afectan a estas poblaciones, incluyendo sus estrategias y decisiones, y, a la vez, contemplando la dimensión histórica (aspecto al cual nos referiremos más adelante). En este sentido, debemos considerar cómo los procesos de expansión de la actividad turística, la "puesta en valor" de la región, los procesos de expansión de la frontera inmobiliaria (asociada al valor de la tierra) y a nuevos agentes foráneos que se fueron radicando en la zona, la privatización de la tierra - que comenzó a darse en la década de 1960 - han incidido en decisiones institucionales (como las de Parques Nacionales) que dieron como resultado los consiguientes desalojos y restricciones a los pobladores y, a raíz de esto, las migraciones hacia las ciudades (acentudas durante aquellos años).

Continuando con la propuesta de Rosberry (1989): "es preciso considerar las otras áreas de las que la gente ha venido [...], las prácticas, tradiciones y valores que los migrantes han traído consigo a una región" (p. 162). En pos de explicar la trayectoria de estas familias del Parque Nacional Huapi, los procesos de emergencia étnica como indígenas y la revalorización comunitaria y territorial que tuvo lugar durante los últimos años, es necesario considerar cómo los migrantes indígenas en ámbitos urbanos toman contacto con diferentes sectores sociales que inciden en la conformación de las organizaciones indígenas en la ciudad. Recordemos que las ciudades - parafraseando a Aravena, Gissi y Toledo (2005) - tienen la particularidad de poner en contacto a conjuntos de residentes de distintas regiones y comunidades y de incentivar el hecho de agruparse como forma de enfrentar la adversidad que supone la migración, lo que 
posibilita el establecimiento de relaciones con instituciones gubernamentales y no gubernamentales, nacionales e internacionales (Aravena et al., 2005).

En la situación específica de Norpatagonia, la presencia de sectores de la Iglesia católica, de migrantes de Chile que por cuestiones políticas - una vez implantada la dictadura militar en dicho país, en 1973- se radicaron en las ciudades argentinas cordilleranas y, por ello, de diferentes organizaciones sociales, políticas y sindicales, es un componente clave para explicar la emergencia, el desarrollo y la consolidación de los movimientos indígenas en la región a partir de la restauración de la democracia. Lo mismo podemos decir de la presencia de migrantes de otras regiones del país que se asentaron en esta zona, ya que Norpatagonia funcionó como una región de "exilio interno" durante la última dictadura cívico-militar.

Por lo tanto, observamos de qué forma las interrelaciones entre los integrantes de las diferentes familias de pobladores con variados sectores sociales inciden en el contexto migratorio urbano para poder visualizar las relaciones que establecen con diferentes sectores sociales y que van a ir delineando e incidiendo en una específica conformación como sujetos sociales, a partir del enriquecimiento de vastas experiencias sociales, políticas y sindicales.

Como señala Bengoa (2009) en relación con la emergencia indígena en las ciudades: "Desde ese nuevo espacio cultural urbano, reinterpretan las viejas culturas comunitarias rurales. Esta es la base de comprensión del fenómeno étnico moderno" (p. 17).

Como antes señalábamos, otro aspecto fundamental es atender a la dimensión histórica. Por ello, hay que retrotraerse a la década de 1930 para visualizar cómo estos pobladores se fueron adaptando a la instalación del Parques Nacionales, o a la década de 1950 para comprender los desalojos forzados y las migraciones a las urbes, y los efectos que estos tuvieron los últimos años sobre el "regreso al territorio". Historicidad que incluye las prácticas, estrategias y transformaciones como resultado del propio accionar de los sujetos. Y también, la reinterpretación efectuada desde el presente. Precisamente, gracias al trabajo efectuado en estos años, contamos con vastos testimonios de la importancia de los recuerdos y la memoria sobre aquellas duras épocas - en ocasiones, trasmitidos de generación en generación- para dar cuenta de las demandas y los reclamos actuales. Como señala Bengoa (2009): "Esa reinterpretación urbana viaja nuevamente al campo y allí se vuelve a reinstalar, reconstruir y rediseñar” (p. 18).

En línea con los tempranos aportes efectuados en nuestro país desde la perspectiva de la antropología social, dedicaremos unas palabras a la importancia que posee la etnografía para dar cuenta de estos procesos a lo largo del tiempo, en pos de vincular las dinámicas específicas o locales con procesos más generales. Paralelamente, para atender a estos últimos, la complementariedad con otras fuentes de información secundaria es clave. En los diferentes testimonios recabados, para explicar el abandono de la actividad agrícolo-ganadera y la migración hacia las incipientes localidades hace cuatro o cinco décadas, aluden a "la muerte de la abuela" (que dirigía y organizaba las tareas) o la "construcción de la ruta" (que impidió que se siguiera cultivando como antes) o "la falta de escuelas" (en el campo), lo que impedía a los pobladores "progresar". Sin embargo, estos testimonios, sumamente valiosos para dar cuenta de dinámicas de comunidades específicas, no nos dicen nada sobre cómo, para la misma época, en diferentes poblaciones de la región, se dieron procesos muy comparables. Los censos u otras fuentes secundarias, ${ }^{15}$ en cambio, nos permiten visualizar estas dinámicas e indagar en torno a estos procesos más regionales que explican el despoblamiento del campo durante aquellos años.
15. A partir de documentos de Parques Nacionales que hemos analizado al momento de la puesta en funciones de esta dependencia en el año 1934 y tres décadas después (1965), observamos una sensible reducción en la cantidad familias para esta última fecha. 
16. Al momento de escribir estas líneas (octubre de 2018), como resultado del ajuste neoliberal, se profundiza la recesión económica, la pérdida del poder adquisitivo por parte de la inmensa mayoría de la población y el crecimiento del desempleo y de la precariedad laboral.
Otra arista fundamental para poder explicar la emergencia de estos años es la importancia que posee el sector doméstico en la trasmisión de normas y valores. Los pobladores, en un contexto urbano y migratorio, luego de varias décadas de haberse establecido en la ciudad (y, en muchos casos, sus descendientes) fueron reconstruyendo su identidad grupal, su pasado y su propia adscripción como mapuches. Entonces, rescatamos también la importancia del grupo doméstico en este rol que trasciende lo económico y lo político.

Para finalizar, deseamos destacar que, en tiempos como en los que vivimos, ${ }^{16}$ de creciente precarización social, se acrecienta la importancia de los ámbitos domésticos como espacios de producción, consumo y reproducción. Por ello, el análisis de esta temática, respecto de la cual procuramos haber efectuado un aporte en estas páginas, constituye una temática fundamental de la antropología económica.

\section{Financiamiento:}

Proyectos de Investigación de la programación UBACYT (Programación 2018) Cod. $20020170100035 B A$ y proyecto Cod. 20020170100294BA. Proyecto de Desarrollo Tecnológico y Social (PDTS) - CONICET, PDTS CIN CONICET Nro. 252, Universidad de Buenos Aires (UBA) y Universidad Nacional del Comahue (UNCo).

\section{Agradecimientos:}

Agradecemos a Pamela Pulcinella por la atenta lectura del texto y sus valiosos aportes.

Universidad de Buenos Aires, Consejo Nacional de Investigaciones Científicas y Técnicas (CONICET), Universidad Nacional del Comahue 


\section{Q Referencias bibliográficas}

» Aravena, A., Gissi, N. y Toledo, G. (2005). Los mapuches más acá y más allá de la frontera: Identidad étnica en las ciudades de Concepción y Temuco. Sociedad Hoy, 8-9, 117-132.

" Archetti, E. y Stølen, K. A. (1975). Explotación familiar y acumulación de capital en el campo argentino. Buenos Aires: Siglo XXI.

"Balazote, A. (2007). Antropología económica y economía política. Córdoba: Centro de Estudios Avanzados, Universidad Nacional de Córdoba.

» Balazote, A. y Radovich, J. C. (1992). El concepto de grupo doméstico. En H. Trinchero (Comp.). Antropología Económica II (pp. 27-43). Buenos Aires: Centro Editor de América Latina.

» Bartolomé, L. (1975). Colonos, plantadores y agroindustrias. La explotación agrícola familiar en el sudeste de Misiones. Desarrollo Económico, 15, 239-264.

» Bartolomé, M. y Barabas, A. (1996). La pluralidad en peligro. Procesos de transfiguración y extinción cultural en Oaxaca: chochos, chontales, ixcatecos y zoques. México: Instituto Nacional Indigenista.

» Bengoa, J. (2009). ¿Una segunda etapa de la Emergencia Indígena en América Latina? Cuadernos de Antropología Social, 29, 7-22.

"Bilbao, S. (1964). Poblamiento y actividad humana en el extremo norte del Chaco santiagueño. Cuadernos del Instituto Nacional de Antropología y Pensamiento Latinoamericano, 5, 143-162.

" Chayanov, A. V. (1974). La organización de la unidad económica campesina. Buenos Aires: Nueva Visión.

"Clastres, P. (1996). Mitos y ritos de los indios de América del Sur. En Investigaciones en antropología política (pp. 65-109). Barcelona: Gedisa.

»Comas D' Argemir, D. (1998). Antropología económica. Barcelona: Ariel.

"Federici, S. (2013). La reproducción de la fuerza de trabajo en la economía global y la inacabada revolución feminista. En Revolución en punto cero. Trabajo doméstico, reproducción y luchas feministas (pp. 153-174). Madrid: Traficantes de sueños.

"Firth, R. (1976). Elementos de Antropología Social. Buenos Aires: Amorrortu Editores.

» Hermitte, E. y Herrán, C. (1977). Sistema productivo, instituciones intersticiales y formas de articulación social en una comunidad del noroeste argentino. En E. Hermitte y L. Bartolomé (Eds.). Procesos de articulación social (pp. 238-256). Buenos Aires: Amorrortu.

» Herrán, C. (1990). Antropología Social en la Argentina: Apuntes y perspectivas. Cuadernos de Antropología Social, 10, 108-114.

"Instituto Nacional de Estadísticas y Censos (INDEC) (2001). Censo Nacional de Población, Hogares y Viviendas 2001, Argentina.

"Instituto Nacional de Estadísticas y Censos (INDEC). (2012). Censo Nacional de Población, Hogares y Viviendas 2010, Censo del Bicentenario. Resultados definitivos, Serie B № 2. Argentina.

» Juliano, M. D. (1996). Los mapuches, la más larga resistencia. Anuario del Instituto de Estudios Histórico Sociales (IEHS), 11, 303-327.

"Meillassoux, C. (1993). Mujeres, graneros y capitales. México: Siglo XXI. 
" Mintz, S. (1996). Dulzura y poder. El lugar del azúcar en la historia moderna. Mexico: Siglo XXI.

» Narotzky, S. (2004). Antropología Económica. Nuevas Tendencias. Barcelona: Melusina.

»Pacheco de Oliveira, J. (Comp.) (1999). Entrando e saindo da "mistura" os indios nos censos nacionais. En Ensaios em Antropologia histórica (pp. 124-151). Río de Janeiro: Editora de la Universidad Federal de Río de Janeiro.

» Radovich, J. C. (1992). Política indígena y movimientos étnicos: el caso Mapuche. Cuadernos de Antropología, 4, 47-65.

» Radovich, J. C. y Balazote, A. (2009). El pueblo mapuche contra la discriminación y el etnocidio. En G. Ghioldi (Comp.). Historia de las familias mapuche Lof Paichil Antriao y Lof Quintriqueo, Mapuche de la margen norte del lago Nahuel Huapi (pp. 35-59). Neuquén: Archivos del Sur, Biblioteca Popular Osvaldo Bayer, Ferreyra editor.

» Ratier, H. (2010). La antropología social argentina: su desarrollo. Antropología y Ciencias Sociales, 9, 17-47.

»Ribeiro, D. (1971). El dilema de América Latina. Estructuras de poder y fuerzas insurgentes. Buenos Aires: Siglo XXI.

"Roseberry, W. (1989). Los campesinos y el mundo. En S. Plattner (Ed.) Antropología económica (pp. 154-176). Ciudad de México: Consejo Nacional para la Cultura y las Artes (CONACULTA).

»Sahlins, M. (1972). Economía de la edad de piedra. Madrid: AKAL.

»Schiavoni, G. (1998). Colonos y ocupantes. Parentesco, reciprocidad y diferenciación social en la frontera agraria de Misiones. Posadas: Editorial Universitaria, Universidad Nacional de Misiones.

"Stoler, A. (1987). Transiciones en Sumatra: el capitalismo colonial y las teorías sobre la subsunción. Revista Internacional de Ciencias Sociales,_XXXIX(39), 4 (114), 543-562.

» Trinchero, H. (Ed.). (1995). Producción doméstica y capital. Estudios desde la Antropología Económica. Buenos Aires: Biblos.

» Trinchero, H., Balazote, A. y Valverde, S. (2007). Antropología Económica y Ecológica: recorridos y desafíos disciplinares. Cuadernos de Antropología Social, 26, 7-19.

»Valverde, S. (2010). Demandas territoriales del pueblo Mapuche en área Parques Nacionales. Avá, 17. - Revista de Antropología, 17, 69-83.

»Valverde, S., García, A. y Bersten, L. (2008). Relatos patagónicos. Historias familiares en la construcción del espacio social en Villa Traful. Córdoba: Ferreyra Editor.

»Vázquez, H. (2000). Procesos identitarios y exclusión sociocultural: la cuestión indígena en la Argentina. Buenos Aires: Biblos.

»Vessuri, H. (1976). La explotación agrícola familiar en el contexto de un sistema de plantación: un caso de la provincia de Tucumán. Desarrollo Económico, 15(58), 215-238.

"Wolf, E. (1971). Los campesinos. Barcelona: Labor. 